\title{
Experimental measurement of oxygen mass transfer and bubble size distribution in an air-water multiphase Taylor-Couette vortex bioreactor
}

\author{
Mahdi Ramezani ${ }^{\mathrm{a}}$, Bo Kong ${ }^{\mathrm{b}}$, Xi Gao ${ }^{\mathrm{b}}$, Michael G. Olsen ${ }^{\mathrm{a}}$, R. Dennis Vigil ${ }^{\mathrm{b}, *}$ \\ ${ }^{a}$ Department of Mechanical Engineering, Iowa State University, Ames, IA, USA \\ ${ }^{\mathrm{b}}$ Department of Chemical and Biological Engineering, Iowa State University, Ames, IA, USA \\ * Corresponding author. E-mail address: vigil@iastate.edu.
}

\begin{abstract}
Experimental measurements of the volumetric liquid mass transfer and bubble size distribution in a vertically oriented semi-batch gas-liquid Taylor-Couette vortex reactor with radius ratio $\eta=r_{i} / r_{o}=0.75$ and aspect ratio $\Gamma=h /\left(r_{o}-r_{i}\right)=40$ were performed, and the results are presented for axial and azimuthal Reynolds number ranges of $R_{a}=11.9-143$ and $\operatorname{Re}_{\Theta}=0-3.5 \times 10^{4}$, respectively. Based on these data, power-law correlations are presented for the dimensionless Sauter mean diameter, bubble size distribution, bubble ellipticity, and volumetric mass transfer coefficient in terms of relevant parameters including the axial and azimuthal Reynolds numbers. The interaction between wall-driven Taylor vortices and the axial passage of buoyancy-driven gas bubbles leads to significantly different dependencies of the mass transfer coefficient on important operating parameters such as inner cylinder angular velocity and axial superficial gas velocity than has been observed in horizontally oriented gas-liquid Taylor vortex reactors. In general, the volumetric mass transfer coefficients in vertical Taylor vortex reactors have a weaker dependence upon both the axial and azimuthal Reynolds numbers and are smaller in magnitude than those observed in horizontal Taylor vortex reactors or in stirred tank reactors. These findings can be explained by differences in the size and spatial distribution of gas bubbles in the vertically oriented reactor in comparison with the other systems.
\end{abstract}

\section{Keywords}

Gas-liquid mass transfer, Sherwood number, Bubble size distribution, Taylor-Couette vortex bioreactor, Multiphase flow 


\section{Nomenclature}

\section{Roman}

C Concentration $[\mathrm{mg} / \mathrm{L}]$

$d \quad$ Bubble diameter [mm]

$D \quad$ Diffusion coefficient $\left[\mathrm{m}^{2} / \mathrm{s}\right]$

E $\quad$ Ellipticity of spheroid

$g \quad$ Gravitational acceleration $\left[\mathrm{m} / \mathrm{s}^{2}\right]$

$h \quad$ Axial distance from the bottom of reactor [m]

$k \quad$ Volumetric mass transfer coefficient $[\mathrm{m} / \mathrm{s}]$

$l \quad$ Spheroid major diameter [m]

$m \quad$ Spheroid minor diameter [m]

$N \quad$ Impeller (inner cylinder) rotational speed [rev/s]

$Q \quad$ Volumetric flow rate $[\mathrm{L} / \mathrm{min}]$

$r_{i} \quad$ Outer radius of the inner cylinder [m]

$r_{o} \quad$ Inner radius of the outer cylinder [m]

$u \quad$ Superficial velocity $[\mathrm{m} / \mathrm{s}]$

Greek

$\Gamma \quad$ Reactor aspect Ratio $\left(\Gamma=h /\left(r_{o}-r_{i}\right)\right)$ 
$\varepsilon_{G} \quad$ Gas holdup

$\eta \quad$ Reactor radius ratio $\left(\eta=r_{i} / r_{o}\right)$

$\omega \quad$ Rotational speed of the inner cylinder [ $\mathrm{rad} / \mathrm{s}]$

$v \quad$ Kinematic viscosity $\left[\mathrm{m}^{2} / \mathrm{s}\right]$

Subscript

a Axial

$b, s \quad$ Bubble surface equivalent

$b, v \quad$ Bubble volume equivalent

$i \quad$ Inner cylinder

G Gas

$L \quad$ Liquid

o $\quad$ Outer cylinder

$s \quad$ Sauter mean

$\Theta \quad$ Azimuthal

Superscript

* Saturation

\section{Introduction}

Gas-liquid mass transfer in agitated vessels is a problem with important industrial applications, and consequently it has been studied extensively, including the development of correlations for volumetric mass transfer coefficients. While most such studies have concentrated on stirred tanks [1-5] or bubble columns [610], relatively few investigations have considered mass transfer in wall-driven gas-liquid flows. In this work, a mass transfer correlation is developed for semi-batch gas-liquid Taylor vortex flow wherein fluid motion is driven by the rotation of a cylinder and by axial passage of buoyant gas bubbles. 
Taylor vortex flow occurs in the annular space between two concentric cylinders, with a rotating inner cylinder and a fixed outer cylinder, as shown in Fig. 1. The flow patterns and instabilities produced in this canonical flow geometry have been extensively studied over the course of many decades [11-22], and Taylor vortex flow patterns have been used in many varied applications such as water purification [23], emulsion polymerization [24,25], liquid-liquid extraction [26,27], pigment preparation [28], photocatalysis [29], culture of animal cells [30], and cultivation of microalgae [31-34]. Nevertheless, despite the sustained and significant attention that Taylor vortex flow has received and the multiphase nature of many of its applications, multiphase TaylorCouette flow remains relatively poorly understood.

The introduction of a second dispersed immiscible fluid phase in Taylor-Couette flow strongly impacts flow patterns and leads to many interesting phenomena. For example, if two immiscible liquids are fed continuously to a horizontally oriented Taylor vortex flow cell, a variety of spatial and spatiotemporal hydrodynamic structures arise [35-38]. More recently it has been reported that the introduction of even a small amount of gas into a vertically oriented Taylor vortex flow cell results in dramatic drag reduction on the rotating inner cylinder and nontrivial gas bubble spatial distribution [39-42]. Some recent studies have been performed in order to characterize these gas-liquid interactions in Taylor-Couette flows [43-46].

However, to our knowledge there exists no previous study of interphase gas-liquid mass transfer in a vertically oriented Taylor-Couette flow device. Dluska et al. [44,47] have investigated gas-liquid mass transfer in a horizontal Taylor vortex reactor with continuous co-flow of both phases. They presented results of both physical and chemical absorption by studying the relationship between the volumetric mass transfer coefficient $k_{L} a$ and the dissipation energy of both axial and azimuthal flow.

In view of the existence of nontrivial flow patterns and phase distribution in a vertically oriented semi-batch gas-liquid Taylor vortex reactor, as well as its potentially important practical applications in biotechnology and other fields, the work presented here describes the development of empirical correlations for bubble size and volumetric interphase mass transfer coefficients. The focus is on high azimuthal Reynolds numbers and gas flow rates that are likely to be relevant to industrial applications. It is shown for fixed reactor geometry (cylinder radii and length) that mass transfer coefficient and droplet diameter depend on both the axial and azimuthal Reynolds numbers. These results are then compared with similar correlations for bubble columns, horizontal Taylor-Couette reactors, and stirred tanks. 


\section{Experimental Methods}

\subsection{Apparatus}

A drawing of the Taylor-Couette apparatus used in this investigation is shown in Fig. 1. The rotating stainless steel inner cylinder has an outer radius of $3.81 \mathrm{~cm}$ and the fixed transparent acrylic outer cylinder has an inner radius of $5.08 \mathrm{~cm}$, resulting in a gap width of $1.27 \mathrm{~cm}$. The length of the reactor is $50.8 \mathrm{~cm}$ and the reactor is filled to a height of $\mathrm{h}=48 \mathrm{~cm}$, hence the total working volume of liquid (deionized water) is $1.70 \mathrm{~L}$. The corresponding radius ratio and aspect ratio of the apparatus are given by $\eta=r_{i} / r_{o}=0.75$ and $\Gamma=h /\left(r_{o}-r_{i}\right)=40$ respectively.

During each experiment the annulus was first filled with room temperature nitrogen-saturated deionized water. Although no specific measures were taken to control the fluid temperature, thermocouple measurements at the top of the reactor showed that after starting the rotation of the inner cylinder and allowing the system to reach a steady state, the working liquid temperature typically fluctuated less than 0.2 degrees Celsius during the duration of an experiment.

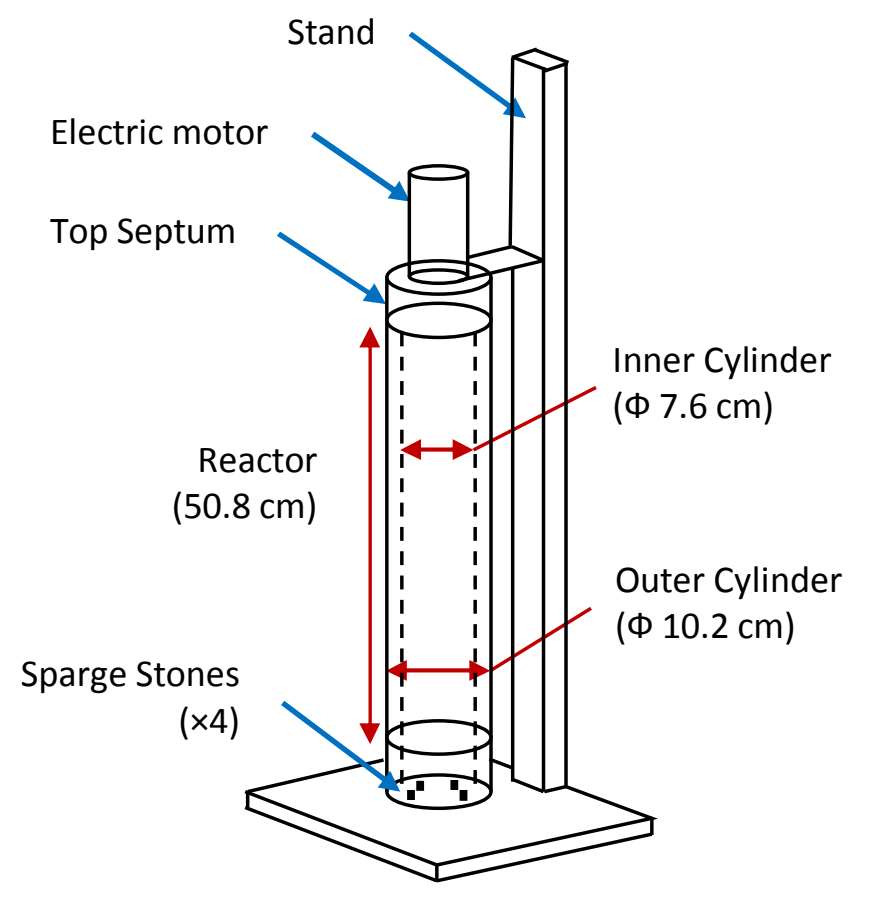

Fig. 1. Taylor-Couette vortex reactor.

The inner cylinder was rotated using a computer-controlled electric motor (Applied Motion STM series) mounted above the apparatus and connected to the inner cylinder with a center slotted-disc flexible shaft 
coupler (McMaster). Rotation rates of zero to $800 \mathrm{rpm}$ with increments of $100 \mathrm{rpm}$ were studied in this experiment, producing azimuthal Reynolds numbers, defined as:

$\operatorname{Re}_{\theta}=\frac{r_{i} \omega_{i}\left(r_{o}-r_{i}\right)}{v_{L}}$

up to $3.5 \times 10^{4}$. For single phase flow, this range is well within the turbulent Taylor vortex flow regime [13].

Gas was continuously fed to the reactor through four equally spaced sparge stones (Sure-Seal Miniature Muffler) mounted in the annular region of the bottom plate. Alicat gas flow rate controllers (MFC series) were used to control both the gas flow rate and the gas mixture composition to within $1 \%$ of set point values. Gases were derived from compressed tanks and premixed before feeding to the reactor. In all experiments, the gas consisted of 79 mole percent nitrogen and 21 mole percent oxygen in order to approximate the composition of air, and to avoid variations in humidity. Total gas volumetric flow rates of $100 \mathrm{sccm}$ to $1200 \mathrm{sccm}$ were studied in this experiment with increments of $200 \mathrm{sccm}$. This corresponds to volumetric flow rates in the range of 0.059 vvm to 0.705 vvm. The axial Reynolds number was defined as:

$\operatorname{Re}_{a}=\frac{2 u_{G}\left(r_{o}-r_{i}\right)}{v_{L}}$

using the superficial gas velocity and the hydraulic diameter of the annulus. Axial Reynolds numbers ranged from 11.9 to 143 , corresponding to superficial gas velocities of $0.47 \mathrm{~mm} / \mathrm{s}$ to $5.64 \mathrm{~mm} / \mathrm{s}$. In the absence of cylinder rotation, these gas velocities correspond to the bubbly flow regime when compared to a bubble column with the same hydraulic diameter [48]. A similar assumption leads to predicted gas holdups of approximately $\varepsilon_{G}$ $=0.03-0.04$ for the highest gas flow rate studied [36]. Over long periods of use and high gas flow rates, heating in the control valves can cause controllers to register unrealistically high gas temperatures, which in turn can affect flow rate accuracy. For this reason the feed gas temperature was carefully monitored during each experiment to ensure that it remained constant, and in the few instances when the gas temperature did not remain constant, the experiment was aborted and the flow controllers were allowed to cool before another run was performed. Each experimental condition considered here was determined uniquely by the rotational speed of the inner cylinder (rpm) and the gas flow rate in vvm, volume gas flow per minute per liquid volume.

\subsection{Bubble Size Measurements}

Several investigators have measured bubble size in gas-liquid reactors $[2,10,49,50]$. One method to measure bubble size is to extract a fluid sample and analyze the bubbles ex situ. Samples taken using this method vary in density, and there is a non-uniform distribution of pressure at the capillary entrance. In addition, the velocity of 
the gas and liquid phases are different, which results in biased selections of bubble size [51]. To avoid these problems, in this work an in-situ optical technique was used to measure bubble sizes. Specifically, digital photographs of the annulus were acquired using a Nikon DSLR camera equipped with an electronic flash and a zoom lens adjusted to a focal length of $31 \mathrm{~mm}$. A shutter speed of 1/500 s and an aperture of f/14 were used to freeze bubble motion and to ensure that the depth of field was sufficient to produce sharp images, an example of which is shown in Fig. 2. A $2 \mathrm{~mm}$ grid printed onto the outer surface of the rotating inner cylinder provided a reference length scale. Although curvature of the transparent outer cylinder results in refraction distortions, the grid provides a means for estimating the severity of this distortion. Bubbles appearing in the middle of each frame were essentially undistorted, and therefore only bubbles appearing in the mid frame region as designated in Fig. 2 were measured.

Because the bubbles are ellipsoidal, bubble size was measured by counting pixels and using the calibration scale to determine major and minor axis lengths for each bubble. Due to the cylindrical shape of the reactor, the scale factors for the horizontal and vertical directions differed by $8 \%$. Since the major and minor axes of the bubbles are inclined in most cases, an average of these two scales was used for all bubble orientations. For each experimental condition studied, 10 photographs were acquired from which at least 150 individual bubbles were measured. Due to the rotational nature of the flow, it was assumed that consecutive images at a fixed axial location were representative of the azimuthal distribution of bubbles, and at least three images were processed for each case. In addition, for every processed image, bubbles from the bottom to the top of the reactor were measured, thereby providing a complete axial sample of bubbles. 

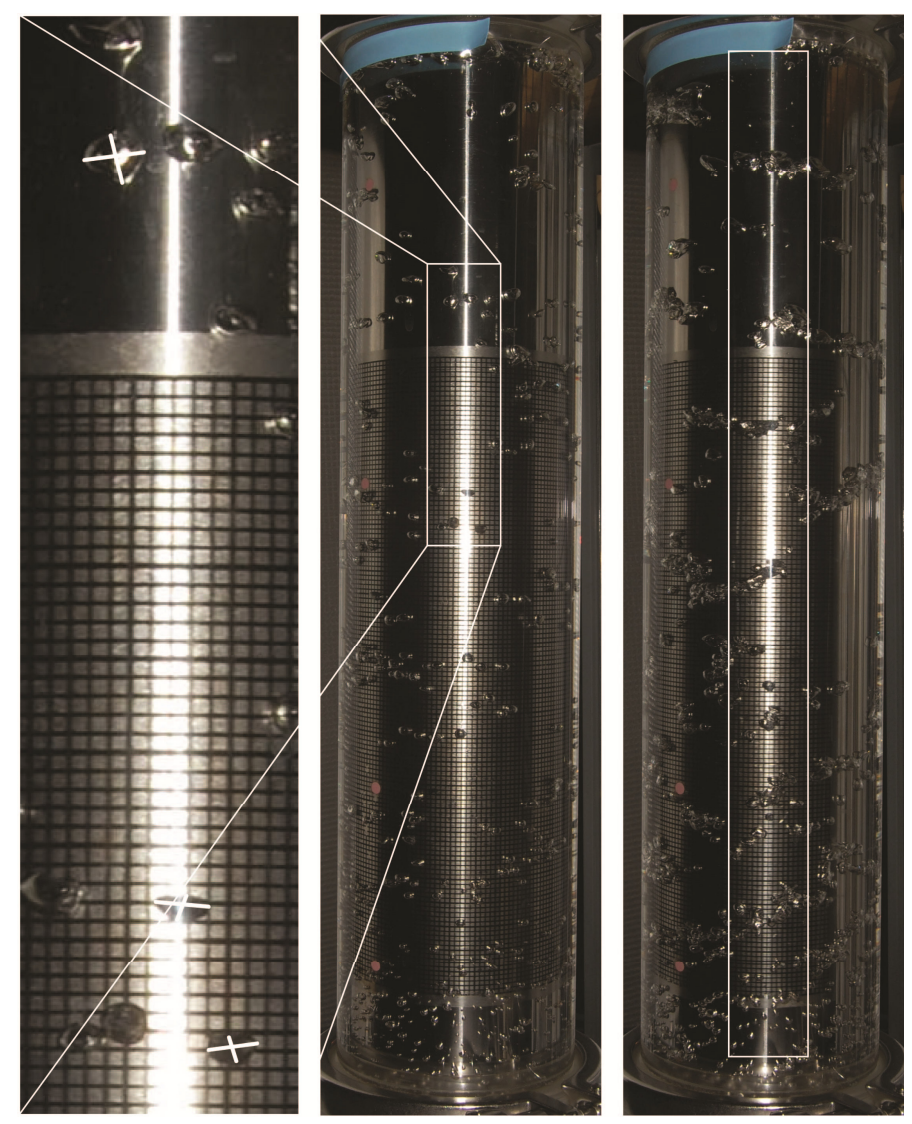

Fig. 2. Sample bubble images including the measuring grid. Left: magnified view of bubble measurement zone I middle: 300 rpm 0.235 vvm I Right: 500 rpm - 0.705 vvm; white rectangle depicts the complete bubble measurement area.

Fig. 2 shows sample bubble images and illustrates the bubble measuring procedure. The photograph in the middle is for a moderate rotation speed and gas flow rate, and individual bubbles can clearly be distinguished. On the left is a magnified portion of the same image showing major and minor axes of several bubbles marked with white lines. For experiments performed with a combination of high rotation speed and gas flow rate, it is nearly impossible to discern individual bubbles due to presence of bubble swarms. The right panel in Fig. 2 shows an image for the highest cylinder rotation speed $(500 \mathrm{rpm})$ and gas flow rate $(0.705 \mathrm{vvm})$ combination investigated for bubble size measurement, and the difficulty in distinguishing individual bubbles is apparent.

\subsection{Mass Transfer Measurements}

The rate of mass transport of oxygen from the gas to the liquid was determined from temporal dissolved oxygen measurements. Specifically, a minimally invasive oxygen sensing system (PreSens Fibox 4 transmitter and Pst3 sensor spots) was used to measure the time-dependent dissolved oxygen concentration in the deionized water at the inside surface of the transparent outer cylinder at several locations along the main axis of the reactor. The 
optical oxygen sensor system employs an external fiber optics laser probe to measure the oxygen concentration at small $5 \mathrm{~mm}$ diameter patches mounted flush with the inside of the stationary outer cylinder wall. In particular, four circular patches of $1 \mathrm{~mm}$ thickness were mounted on the inside surface at different axial distances of $5.1 \mathrm{~cm}, 12.7 \mathrm{~cm}, 25.4 \mathrm{~cm}$, and $38.1 \mathrm{~cm}$ measured from the bottom of the reactor. The small size and thickness of these patches make them practically a non-intrusive means of measurement. The accuracy of the measured concentrations using this instrument is $\pm 3 \%$ for dissolved oxygen. The probe response time is less than 6 seconds, while the shortest mass transfer response time $\left(1 / k_{L} a\right)$ measured was approximately 50 seconds.

Oxygen mass transfer experiments were performed using a dynamic method based on the measurement of the concentration of dissolved oxygen in water [52]. A step change in the concentration of inlet gas results in a dynamic response in the concentration of oxygen dissolved in the liquid phase, and these temporal concentration data are used to calculate a mass transfer coefficient. Experiments were performed for a gas flow rate range of $0.059 \mathrm{vvm}$ to $0.705 \mathrm{vvm}$ and rotation rates of up to $800 \mathrm{rpm}$, corresponding to axial Reynolds number ranging between 11.9 and 143 and azimuthal Reynolds numbers up to $3.5 \times 10^{4}$, respectively.

In a typical experiment, the annular reactor was filled with room temperature deionized water. Use of deionized water is important due to the fact that the presence of ions in water can reduce the average bubble size, which results in a larger specific interfacial area $(a)$ [51]. Subsequently, pure nitrogen gas was pumped through sparge stones (using a separate gas flow controller than the ones used for the simulated air) into the bottom of the reactor at a volumetric flow rate of $512 \mathrm{sccm}$ without rotating the inner cylinder. During this oxygen displacement stage the dissolved oxygen concentration was monitored, and after it reached a minimum value of $0.15 \mathrm{mg} / \mathrm{L}$, the flow of nitrogen was stopped and 5 minutes of rest time was elapsed to ensure that nitrogen bubbles exited the reactor.

After executing the oxygen purge procedure described above, the inner cylinder was accelerated to the desired rotation speed and flow of simulated air commenced at the chosen volumetric flow rate. The dissolved oxygen concentration was continually monitored throughout this process until it returned to the initial value (approximately $8 \mathrm{mg} / \mathrm{L}$ ) measured before the oxygen purge began. Because the fluid flow does not respond instantaneously to the step changes in gas flow rate and cylinder rotation speed, the dissolved oxygen measurements obtained during the first 30 seconds after the simulated air is introduced into the reactor were omitted from calculations of the volumetric mass transfer coefficient.

A typical plot of the dissolved oxygen concentration versus time during the three steps described above (oxygen purge, no gas feed, and feed of simulated air) is shown in Fig. 3. Also indicated is the portion of the data that 
was used for mass transfer calculations described in section 3.1. At the end of data collection for a given experimental trial, the water was replaced before repeating the protocol described above for the next trial.

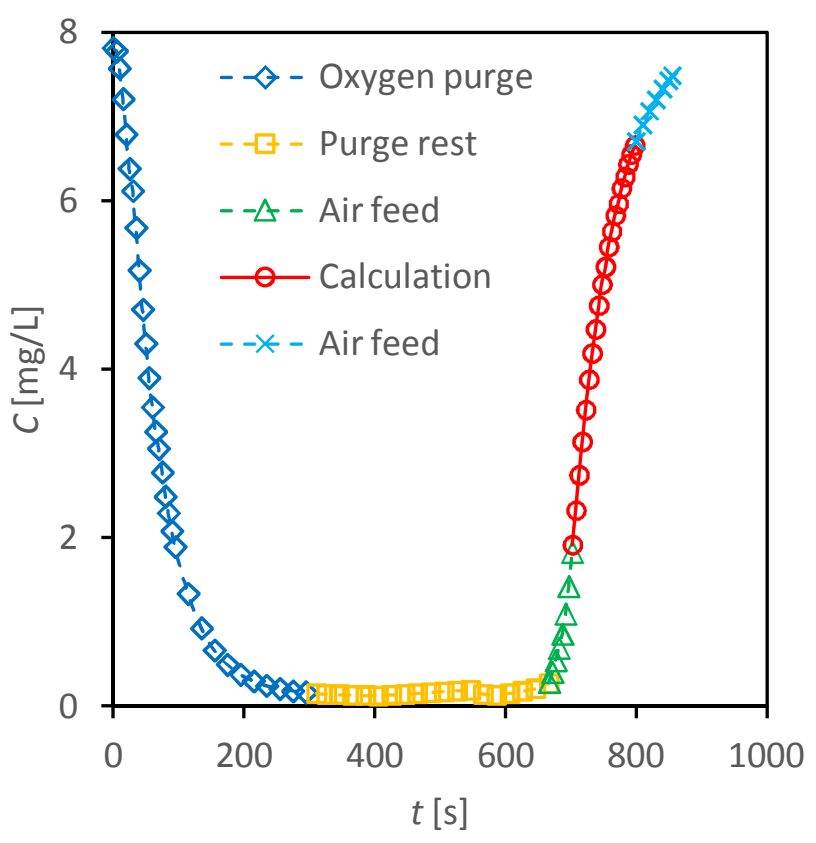

Fig. 3. A full cycle of oxygen concentration data collection, showing the portion used for mass transfer calculations.

An attempt was made to quantify the variation of the mass transfer coefficient with reactor axial position by measuring $k_{L} a$ at four different axial locations for the five cylinder rotation/gas flow rate combinations of $(0$ rpm, $0.059 \mathrm{vvm}),(100 \mathrm{rpm}, 0.470 \mathrm{vvm}),(200 \mathrm{rpm}, 0.117 \mathrm{vvm}),(300 \mathrm{rpm}, 0.117 \mathrm{vvm})$, and (400 rpm, 0.352 $\mathrm{vVm}$ ). It was observed from these experiments that the variation of $k_{L} a$ with axial position, with the exception of the location closest to gas inlet, was smaller than the accuracy of the measurements at a fixed axial position. For this reason, no further experiments were carried out at multiple axial positions and instead the remaining data were collected at an axial position of $12.7 \mathrm{~cm}$ above the gas feed, and these data were deemed sufficient to compute representative average reactor mass transfer coefficients.

\section{Calculations}

\subsection{Bubble Size}

It has been previously observed that for buoyancy-driven flows in the absence of agitation (for example in bubble columns), bubbles take on the shape of oblate spheroids [50,53]. In such cases the bubble minor axis is aligned with the principle direction of their motion. In contrast, in the present case bubble motion is driven both by fluid shear generated by the rotating inner cylinder and by buoyancy. Even for the lowest azimuthal 
Reynolds numbers investigated, the azimuthal motion of bubbles is significant when compared to the axial distance of travel. Consequently, it is not surprising that photographs of bubbles in the Taylor vortex reactor show that bubble major axes are nearly horizontal. Furthermore, because the sheared bubbles have a distinctly ellipsoidal appearance with the major axis aligned horizontally with the azimuthal component of velocity, it is more appropriate to approximate their shapes as prolate spheroids rather than oblate spheroids, except in the case of no cylinder rotation. The recent work of Qiao et al. [54] also suggests that bubbles are stretched circumferentially in Taylor-Couette vortex flow, and at least one other investigation reported prolate bubbles in a vertical Taylor Couette reactor [40].

In view of the above discussion and for the purpose of calculating bubble sizes, bubbles were assumed to be oblate in the absence of rotation of the inner cylinder whereas they were assumed to be prolate for cases with cylinder rotation. Specifically, the volume equivalent bubble diameter was computed using Eqs. (3) and (4) for oblate and prolate spheroids, respectively.

$d_{b, v}^{\text {prolate }}=\sqrt[3]{l m^{2}}$

$d_{b, v}^{\text {oblate }}=\sqrt[3]{l^{2} m}$

where $l$ is the major diameter of the spheroid and $m$ is the minor diameter. Subsequently, the Sauter mean diameter for a population of bubbles is obtained from Eq. (5) [48,55].

$d_{s, v}=\sum d_{b, v}^{3} / \sum d_{b, v}^{2}$

The above approach for computing the Sauter mean diameter assumes that bubble elongation, as determined by the ratio of the major to minor axis, is modest. However for non-spheroidal bubbles a more elaborate calculation of bubble diameter is possible as suggested by Muroyama et al. [56,57]. Since in all cases studied here bubble elongation was significant (with maximum aspect ratios typically ranging from 2.5-4.0), the latter method was employed. This method requires calculation of the surface equivalent diameter using Eqs. (6) and (7), assuming prolate or oblate spheroids respectively [58].

$d_{b, s}^{\text {prolate }}=\sqrt{1 / 2\left(m^{2}+l m / E \sin ^{-1} E\right)}$ 
$d_{b, s}^{\text {oblate }}=\sqrt{1 / 2\left(l^{2}+m^{2} / 2 E^{\left.\ln \left(\frac{1+E}{1-E}\right)\right)}\right.}$

Here, the ellipticity of a spheroid is calculated using:

$E=\sqrt{1-\frac{m^{2}}{l^{2}}}$

where $E$ is equal to one for the case of a sphere. Based on these definitions, it is possible to calculate a Sauter diameter for each individual bubble defined as:

$d_{s}=d_{b, v}^{3} / d_{b, s}^{2}$

These Sauter diameters were used for the calculation of the mean and standard deviation for the bubble size distribution presented in section 4. However, for the calculation of the Sherwood number, a Sauter mean diameter is used to represent the average bubble diameter, defined by:

$d_{32}=\sum d_{b, v}^{3} / \sum d_{b, s}^{2}$

It is important to note that the mean of the bubble Sauter diameters calculated using Eq. (9) is different from the Sauter mean of bubble diameters calculated using Eq. (10). Table 1 presents a comparison between the different definitions of bubble diameters given here for the case of $300 \mathrm{rpm}$ and $0.470 \mathrm{vvm}$ from a sample of 151 measured bubble diameters. The brackets denote sample means. The case reported in Table 1 has a mean ellipticity of 0.660 and minimum and maximum ellipticities of 0.035 and 0.964 , respectively. These same values correspond to a mean of 1.47 , minimum of 1.00 and a maximum of 3.75 for the bubble aspect ratio, which is simply the ratio of the major diameter over minor diameter $(\mathrm{l} / \mathrm{m})$.

Table 1. Comparison of mean bubble diameter values using different definitions, $300 \mathrm{rpm}-0.470 \mathrm{vvm}$. All values are in mm.

$\begin{array}{clll}\text { Definition } & \begin{array}{l}\text { Prolate } \\ \text { spheroid }\end{array} & \begin{array}{l}\text { Oblate } \\ \text { Spheroid }\end{array} & \text { Equation } \\ \left\langle\boldsymbol{d}_{\boldsymbol{b}, \boldsymbol{v}}\right\rangle & 3.27 & 3.70 & 3,4 \\ \left\langle\boldsymbol{d}_{\boldsymbol{b}, \boldsymbol{s}}\right\rangle & 3.32 & 3.77 & 6,7 \\ \left\langle\boldsymbol{d}_{\boldsymbol{s}}\right\rangle & 3.19 & 3.57 & 9\end{array}$




$\begin{array}{llll}\boldsymbol{\sigma}_{\boldsymbol{d}_{\boldsymbol{s}}} & 0.94 & 1.05 & 9 \\ \boldsymbol{d}_{\boldsymbol{s}, \boldsymbol{v}} & 3.87 & 4.41 & 5 \\ \boldsymbol{d}_{32} & 3.76 & 4.24 & 10\end{array}$

Table 1 demonstrates a significant difference in computed diameters depending upon whether bubbles are assumed to be prolate or oblate spheroids. Relatively smaller difference in the Sauter mean between prolate and oblate spheroids occurs using Eq. (10) rather than Eq. (5). It is worth noting that the mean of the Sauter diameter calculated using Eq. (9) is smaller than the Sauter mean diameter calculated using either Eq. (5) or Eq. (10).

\subsection{Mass Transfer Coefficient}

In order to compute a volumetric mass transfer coefficient based upon a time series of dissolved oxygen concentrations, it is necessary to invoke an approximation concerning the mixing of the liquid phase.

Concentration measurements of dissolved oxygen were performed at four different axial positions and the mass transfer coefficient was found to be independent of axial position within the accuracy of the measurement method used in this study and accordingly, the liquid phase was assumed to be well-mixed. Hence, an oxygen mole balance on the liquid phase (assuming that no oxygen is dissolved in the liquid initially) leads to:

$\operatorname{Ln}\left(1-\frac{c_{L}}{C^{*}}\right)=-k_{L} a t$

where $C^{*}$ and $C_{L}$ are the oxygen concentrations in the liquid for saturated conditions and at an arbitrary time $t$, respectively, $a$ is the specific gas-liquid interfacial surface area, and $k_{L}$ is the liquid volumetric mass transfer coefficient [52]. It should be mentioned that although the initial concentration of oxygen is measured for each experiment, assuming a value of zero (instead of the measured initial oxygen concentration) does not change the computed value of $k_{L} a$ and therefore Eq. (11) may be used without modification. However, this equation does require an accurate value for the saturation concentration $C^{*}$, which depends upon temperature. The temperature dependence of the saturation concentration was determined using the correlation of Battino et al. [59]:

$C^{*}=\exp (-52.16764+84.59929 / \tau+23.41230 \ln \tau)$

where $\tau=T / 100 \mathrm{~K}$, the temperature is in Kelvins and $C^{*}$ has units of $\mathrm{mL}_{\text {(Gas) }} / \mathrm{L}_{\text {(Liquid). }}$.

A typical plot of experimental data according to Eq. (11) for five different cases is depicted in Fig. 4. 
Over a relatively long range of time the plots are linear, suggesting that the well-mixed approximation is justified. Hence, by fitting a line to the linear portion of the curve, $k_{L} a$ can be estimated from the slope. In all cases studied in this work the linear fit resulted in a normalized squared residual of 0.995 or greater.

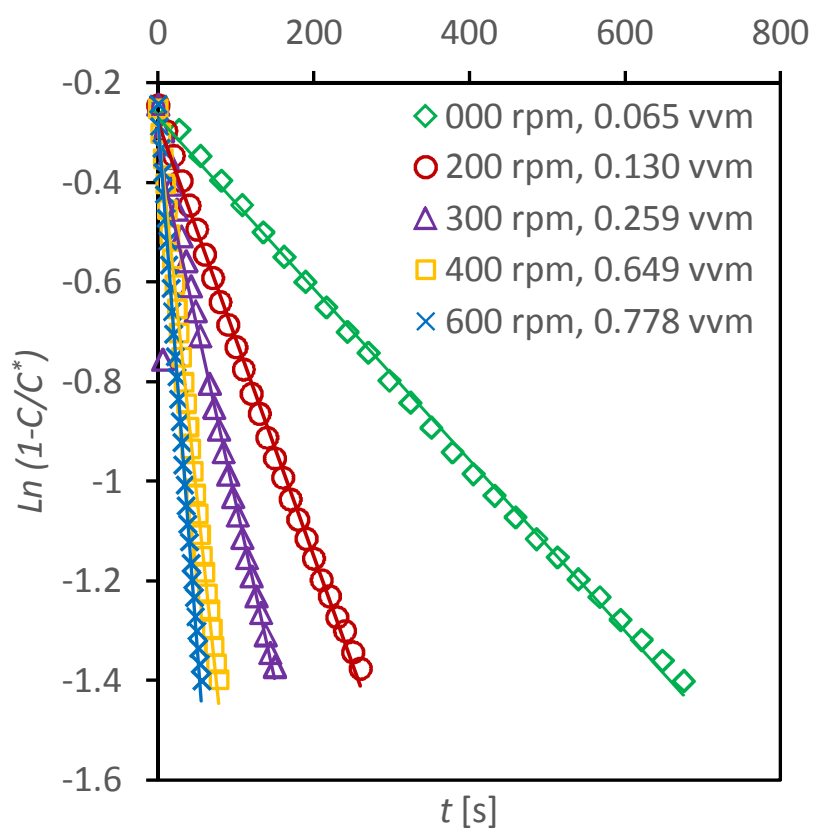

Fig. 4. Sample linear plot for calculation of $k_{L} a$ for five of the cases studied in this work.

\section{Results and Discussion}

\subsection{Bubble Size}

The Sauter mean bubble diameter is reported in Table 2 for all cases for which measurements were acquired. The two missing entries in this table are a result of experimental conditions that produce bubble swarms, thereby making it impossible to distinguish individual bubbles.

Table 2. Sauter mean bubble diameters measured for different cases.

\begin{tabular}{|c|c|c|c|c|}
\hline$r p$ & 0 & 100 & 300 & 500 \\
\hline 0.059 & 2.12 & 2.24 & 3.28 & 3.82 \\
\hline 0.235 & 2.26 & 2.69 & 3.38 & 4.06 \\
\hline 0.470 & 2.91 & 3.42 & 3.76 & \\
\hline 0.705 & 3.11 & 3.06 & 4.11 & \\
\hline
\end{tabular}


Individual measured major and minor axis dimensions are plotted in Fig. 5 for experiments carried out with no inner cylinder rotation. For comparison, the data of Akita and Yoshida [49] obtained using a bubble column are also shown. However the gas flow rate for which those investigators obtained their data was not reported. With this caveat, it is apparent from Fig. 5 that the oblate gas bubbles observed in the annular geometry are more ellipsoidal than those found in a cylindrical bubble column. Evidently, wall effects associated with the annular flow result in greater bubble distortion than in the cylindrical bubble column.

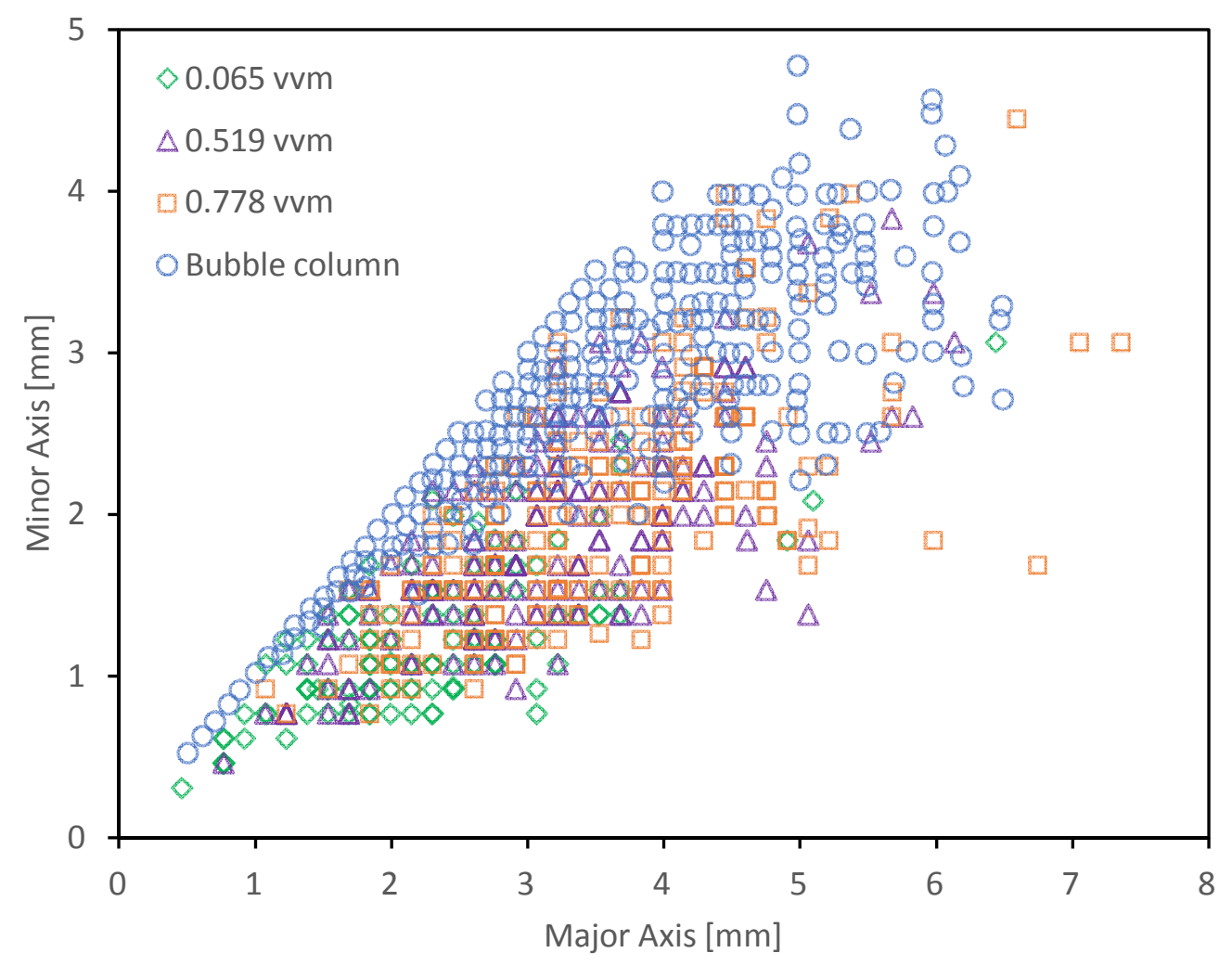

Fig. 5. Comparison of individual measured bubble dimensions for non-rotating cases against bubble column data [49].

In order to generalize these results, a bubble diameter correlation was developed based upon the two variables considered, namely the cylinder rotation speed and the gas flow rate. The effect of the wall-driven flow can be represented by an azimuthal Reynolds number, defined by Eq. (1). Similarly, the strength of the axial gas flow can be quantified by the axial Reynolds number as defined in Eq. (2).

Although the bubble diameter should depend upon the superficial gas velocity, it can be expected that other factors, such as the bubble buoyancy and surface tension also play important roles. However, in this study a single pair of fluids was used, and therefore the densities of the fluids and the interfacial surface tension were 
not varied. Hence, in developing a correlation for the mean bubble diameter, other factors that may be important for a more general correlation were omitted, such as the Bond number. Nevertheless, since aqueous solutions in contact with low molecular weight gases are common, the correlations developed here may have wide applicability.

Here it is assumed that the Sauter mean diameter can be correlated with the azimuthal and axial Reynolds numbers using a power law relation. The correlation also ensures that the bubble size has a non-zero value at zero azimuthal Reynolds number. The following expression was then obtained using nonlinear least squares regression.

$\frac{d_{32}}{r_{o}-r_{i}}=1.0 \times 10^{-5} \operatorname{Re}_{a}^{0.11}\left(\operatorname{Re}_{\theta}+3.3 \times 10^{4}\right)^{0.91}$

A comparison of the dimensionless bubble diameters predicted by this correlation versus experimentally measured values is presented in supplementary material Fig. S1, which demonstrates close agreement quantified by a normalized $\mathrm{R}^{2}$ value of 0.983 . For small azimuthal Reynolds numbers, the constant in parenthesis dominates, and the bubble size is relatively independent of the rotational speed of the inner cylinder. For larger azimuthal Reynolds numbers, the monotonic increase in bubble size can be explained as follows. It was observed that as the inner cylinder rotation speed increases, the gas bubbles form bands at boundaries between Taylor vortices, similar to those observed by Hubacz and Wronski [43]. In addition, although gas holdup was not measured, our visual observations are consistent with the hypothesis that gas holdup increases with increasing rotational speed. As a result of these two factors (increased bubble proximity and gas holdup), it can be expected that bubble size will increase due to bubble coalescence as the azimuthal Reynolds number increases.

Detailed simulations of bubbly flow in a Taylor-Couette reactor may require a distribution of bubble sizes rather than simply the Sauter mean diameter. The distributions of bubble sizes calculated using Eq. (9) for five experimental cases are shown in Fig. 6, and each distribution is fit to a log-normal probability density function having identical values of the mean and standard deviation as the corresponding experimental distribution. In all cases, the experimentally determined bubble size distributions are well represented by log-normal functions. 


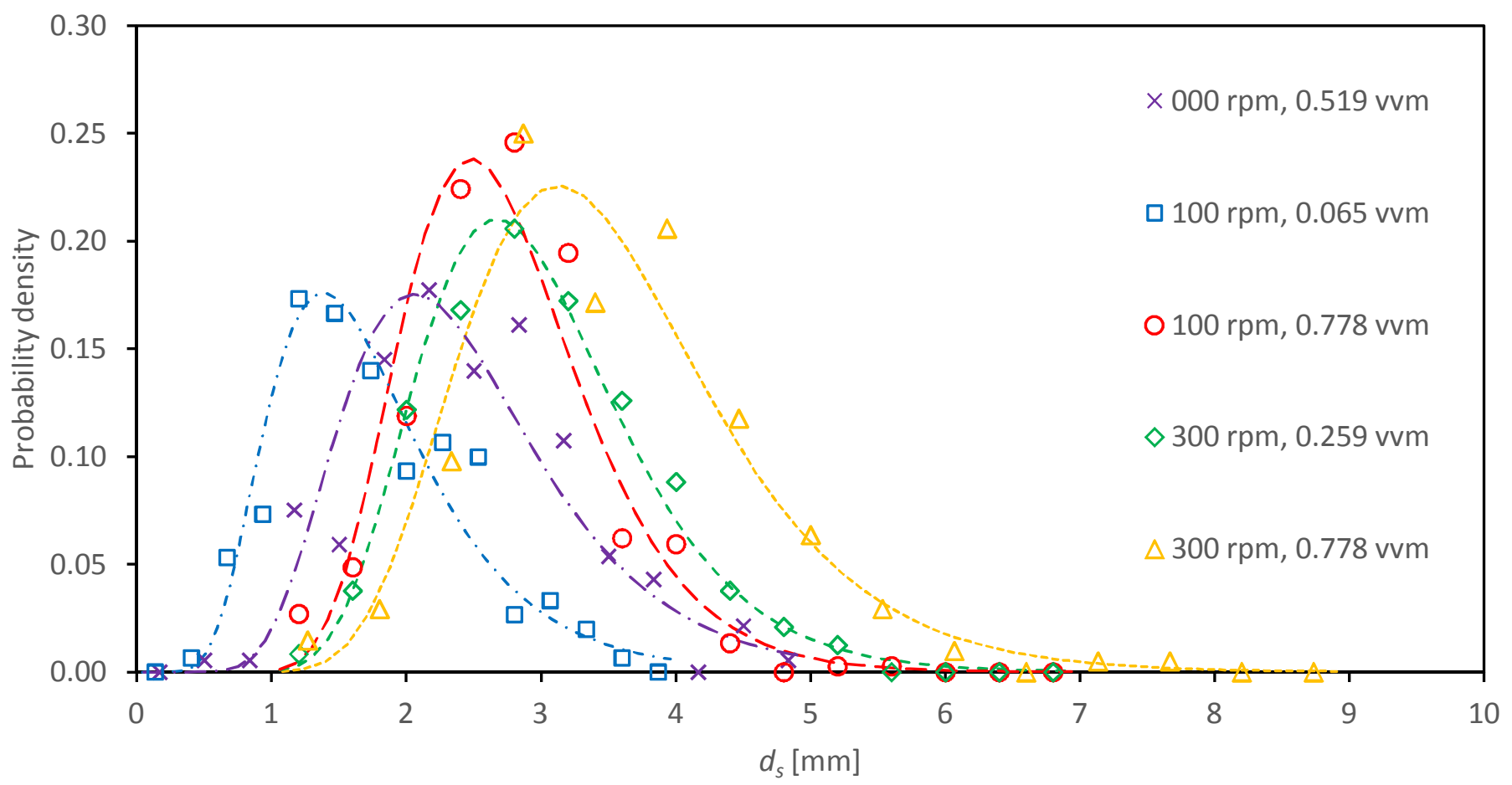

Fig. 6. Probability density function of the Sauter diameter of bubbles $\left(d_{s}\right)$ using Eq. (9) and comparison against log-normal distribution.

Following the same approach as for the Sauter mean diameter, a correlation was developed for calculating the mean value of the log-normal bubble size distribution, resulting in the following equation:

$\mu_{d_{s}}=3.6 \times 10^{-6} \operatorname{Re}_{a}^{0.15}\left(\operatorname{Re}_{\theta}+2.7 \times 10^{4}\right)^{0.89}$

which results in a normalized $\mathrm{R}^{2}$ value of 0.963 .

The standard deviation of the size distributions was approximately $\sigma_{d_{s}}=0.31 \pm 0.08$ for all cases considered, and did not appear to obey any trend with respect to the independent parameters. In addition, the average ellipticity of the bubbles was $\langle E\rangle=0.72 \pm 0.12$.

\subsection{Mass Transfer Coefficient}

Values of $k_{L} a$ were obtained from dissolved oxygen measurements described in section 2.3, and these results are shown in Fig. 7 for various cylinder rotation speeds and gas flow rates. In four instances, experiments were repeated two times in order to determine variability of the data. As a result of these replications, the data represented in this study are estimated to be reproducible within a deviation of $\pm 6 \%$. Rather than performing 
experiments for every possible combination of axial and azimuthal Reynolds numbers, approximately $63 \%$ of the cases were studied.

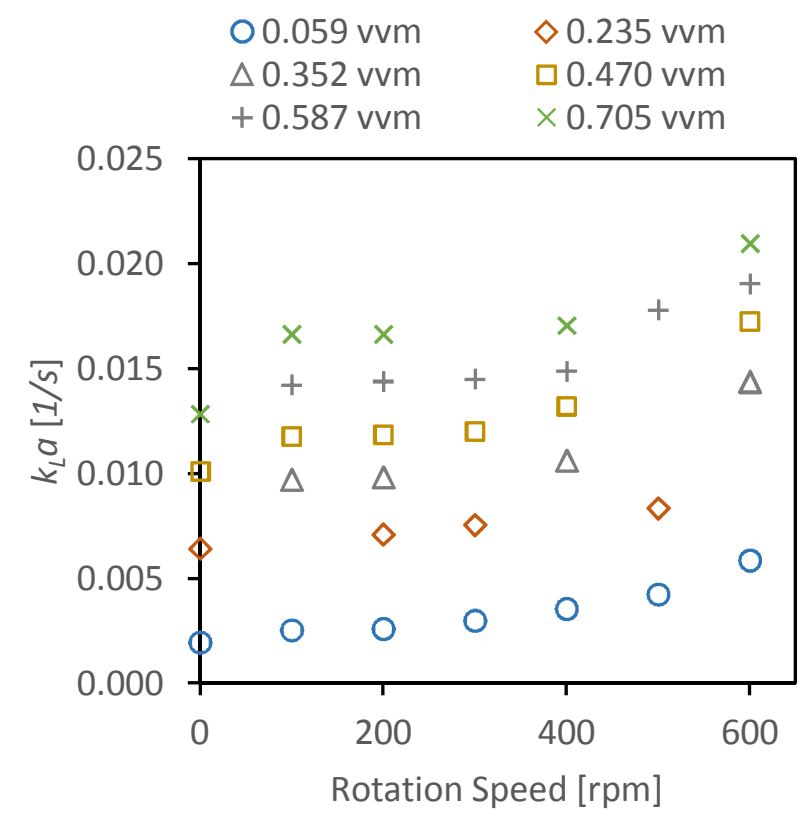

Fig. 7 Calculated values of $\mathrm{k}_{\mathrm{L}}$ a for various rotation speeds at different gas flow rates.

It can be seen from Fig. 7 that for the higher gas flow rates studied, the mass transfer coefficient at low rotation speeds is significantly greater than the value found with no rotation. However, the value of $k_{L} a$ appears to be insensitive to rotation speeds up to approximately $300 \mathrm{rpm}$, irrespective of the gas flow rate. Above rotation speeds of $400 \mathrm{rpm}$, the mass transfer coefficient increases approximately linearly. It is worth noting that the bubble size increases steadily at all the rotation speeds studied.

The dependence of the volumetric mass transfer coefficient on gas flow rate is shown in Fig. 8. For purposes of comparison, the correlation proposed by Heijnen and Van't Riet [7] for bubble columns is also shown in Fig. 8. Except for the highest rotation speed considered, the mass transfer coefficient measured in the Taylor-Couette device is twice as large as those predicted for a bubble column. The departure of the data for the rotation rate of $600 \mathrm{rpm}$ is most likely due to coalescence of bubbles as discussed in section 4.1. 


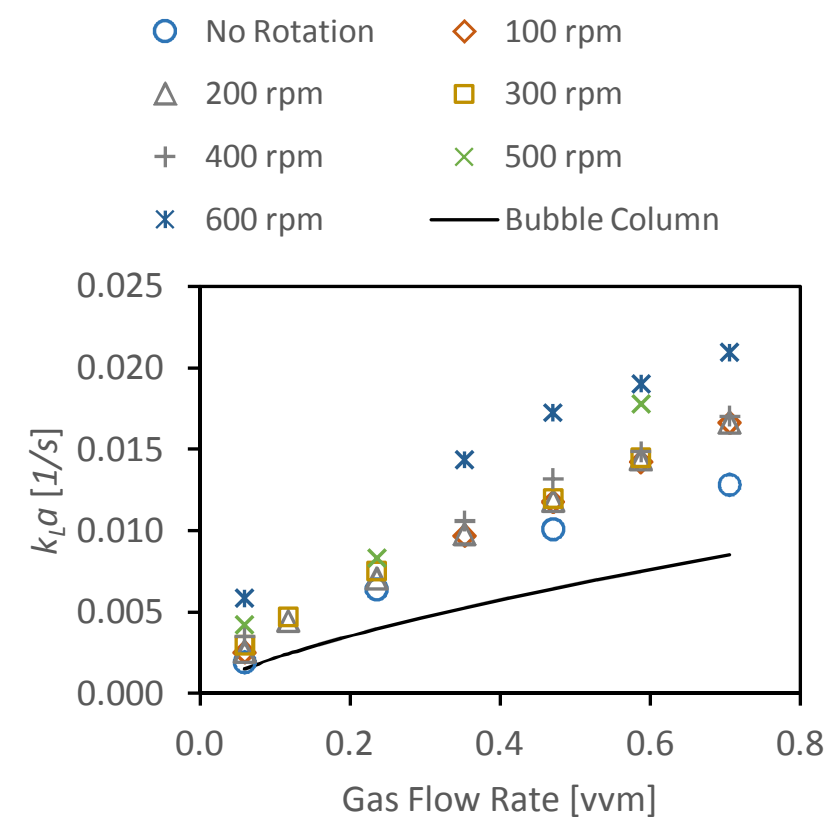

Fig. 8 Calculated values of $k_{L} a$ for various gas flow rates at different rotation speeds. Comparison to a bubble column [7].

Direct measurement of the gas holdup, and thus the specific interfacial surface area, was not performed due to the complicated motion of the water free surface in the reactor caused by the rotation of the inner cylinder and the escaping gas. In order to cope with this limitation, the following definition of the modified Sherwood number based on the Sauter mean diameter of bubbles was used in this study [60,61].

$\mathrm{Sh}=\frac{k_{L} a d_{32}^{2}}{D_{L}}$

The diffusion coefficient of oxygen in water is $D_{L}=2.1 \times 10^{-9} \mathrm{~m}^{2} / \mathrm{s}$ for the average room temperature at which experiments were performed. For cases with a rotating inner cylinder, it is reasonable to assume that the Sherwood number will depend upon the strength of the wall-driven Taylor vortex flow (characterized by the azimuthal Reynolds number) as well as the strength of the axial gas flow (characterized by the axial Reynolds number). As discussed previously, a more general correlation accounting for buoyancy and surface tension would also include other factors such as the Bond number, but since these factors were not considered, they are omitted here. It should also be noted that the bubble size correlation of Eq. (13) was used to compute the Sherwood number. The mass transfer correlation resulting from nonlinear least squares regression is given by:

$\mathrm{Sh}=1.45 \times 10^{-7} \operatorname{Re}_{a}^{0.82}\left(\operatorname{Re}_{\theta}+1.47 \times 10^{4}\right)^{1.61}$ 
The values of the Sherwood number predicted by this equation are compared to values calculated from experimental measurements in supplementary material Fig. $\mathrm{S} 2$, and the normalized $\mathrm{R}^{2}$ is 0.984 , thereby demonstrating an excellent fit.

It can be seen from the proposed correlation that at low rotation speeds, the term containing the azimuthal Reynolds number is dominated by the constant in parenthesis, and consequently mass transfer coefficients are predicted to be insensitive to cylinder rotation speed for small azimuthal Reynolds numbers. In contrast, an increase in the value of the axial Reynolds number results in monotonic growth in the value of Sherwood number.

Fig. 9 shows a comparison of the volumetric mass transfer data of the current study with those published by Dluska et al. [47] for a similar range of axial Reynolds numbers. It is important to note that their experiments were performed using a horizontal Taylor-Couette system with co-flow of liquid and gas phases, using various ratios of the volumetric flow rates of the gas and liquid phases. In contrast, in the present study there is no net axial flow of the liquid phase and buoyancy can be expected to play an important role in driving the axial component of the gas bubble velocity. Here the axial Reynolds numbers are calculated based on the superficial gas velocity according to Eq. (2) and the superficial liquid velocity is neglected. Therefore, direct comparison of axial Reynolds numbers used in the two systems is not possible, and it should be observed that the difference between the superficial gas and liquid velocities, $u_{G}-u_{L}$, in the system with co-flow can be negative, positive or zero whereas it is always positive for the semi-batch system. Nevertheless, Fig. 9 demonstrates that interphase mass transfer is significantly enhanced by rotation of the inner cylinder for a horizontal orientation with co-flow of the two phases, whereas mass transfer is relatively insensitive to cylinder rotation speed for the vertical reactor orientation and semi-batch operation. With no inner cylinder rotation, the mass transfer coefficients in the vertical and horizontal reactors are similar irrespective of the axial flow conditions used.

The difference in the dependence of interphase mass transfer coefficients for the two reactors and flow configurations may be explained as follows. In the horizontal co-flow configuration at sufficiently low cylinder rotation speed, the gas will not be uniformly distributed azimuthally due to the strong relative contribution of buoyancy, and hence gas accumulates at the azimuthal location corresponding to the highest vertical location in the gravity field. Higher cylinder rotation speeds result in greater azimuthal (and radial) dispersion of the gas as well as smaller bubble sizes [43]. As a result of this dispersion and breakup of the gas phase into smaller bubbles, interfacial surface area increases as the azimuthal Reynolds number increases and it can be observed that the increase in the value of $k_{L} a$ is merely due to the increase of the interfacial area [44]. It is shown that the 
value of the mass transfer coefficient stays relatively constant for the horizontal reactor above a threshold of rotation speed that corresponds to break up of the large gas regions into smaller individual bubbles [45].

Furthermore, in the horizontal configuration the buoyant force has no axial component and only acts in the radial and azimuthal directions, leading to higher bubble slip velocities where the buoyant force opposes fluid motion. In contrast, as reported in section 4.1, bubble sizes increase with an increase in azimuthal Reynolds number in the vertical configuration. In addition the bubble dynamics are very different since the buoyant force acts only in the axial direction in the vertical reactor. As discussed in section 4.1, increases in the cylinder rotation speed lead to radial bubble migration towards the inner cylinder and between the Taylor vortices and bubble size increases. Due to this effect, the change in the interfacial area in the vertical reactor as a result of higher rotation speeds is expected to be fairly negligible comparing to the horizontal reactor.

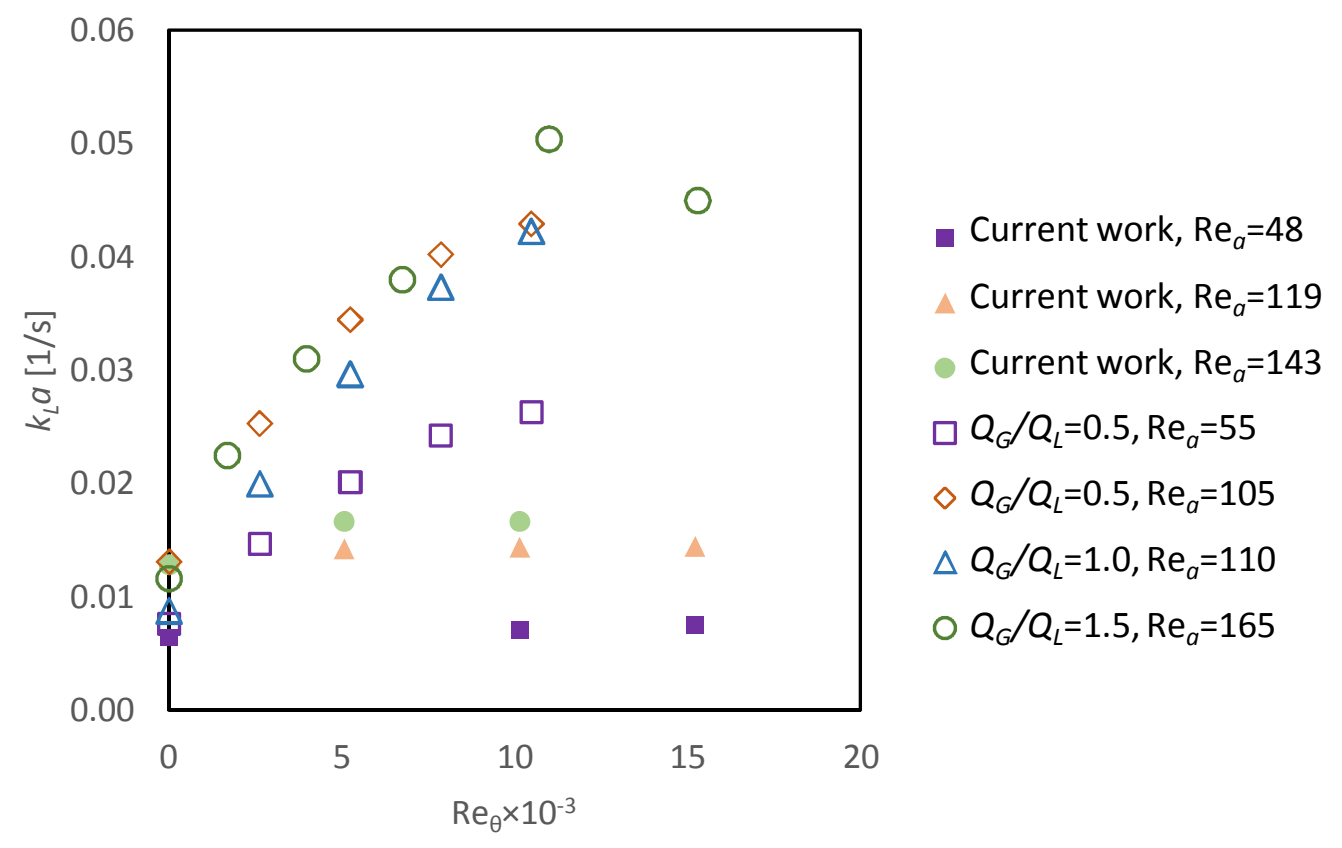

Fig. 9. Volumetric mass transfer coefficient versus azimuthal Reynolds number at moderate to high axial Reynolds numbers compared with data for a horizontal Taylor-Couette reactor with co-flow of phases [47].

It is also useful to compare interphase mass transfer coefficients in a semi-batch vertical Taylor-Couette vortex reactor with those observed in stirred tanks. For example, the correlation of Kapic and Heindel [3] implies the following relation:

$k_{L} a \propto u_{G}^{0.93} N^{1.342}$ 
This type of function inherently assumes the mass transfer coefficient to be zero when the impeller is stopped which in the correct case, it needs to converge to the bubble column value. In order to make the comparison possible, using data obtained in the present study excluding the non-rotating cases, an analogous correlation can be developed for the volumetric mass transfer coefficient in terms of parameters corresponding to those in Eq. (17) by equating the impeller diameter in a stirred tank with the inner cylinder diameter of a Taylor vortex reactor as follows:

$k_{L} a=0.423 u_{G}^{0.668} N^{0.178}$

This correlation has a normalized $\mathrm{R}^{2}$ value of 0.981 , which is an excellent fit within the range of the parameters studied. Comparison of exponents in Eqs. (17) and (18) gives insight into differences between the two types of reactors. Specifically, volumetric mass transfer coefficients in stirred tanks are more sensitive to both superficial gas velocity and impeller speed. In contrast, the generation of fluid shear through the motion of a long smooth wall (as opposed to an impeller) results in formation of Taylor vortices, large scale flow structures that efficiently transport energy and momentum towards the outer wall with relatively small variation in local shear rate, as compared to a stirred tank. This characteristic, namely the relatively uniform shear environment in a Taylor vortex reactor, is highly advantageous for processing material that may be susceptible to shear stress damage, such as microorganisms. However, much greater rotation speeds (as compared to the impeller in a stirred tank) are then required to distribute energy to smaller length scales that are efficacious for increasing interphase mass transport via bubble size reduction and increase of bubble slip velocity.

In the absence of inner cylinder rotation, the Taylor vortex reactor becomes an annular bubble column, and therefore it is useful to consider comparison of the correlations developed here (for $\operatorname{Re}_{\theta}=0$ ) with those available for bubble columns. Furthermore, because the rise of the free surface location (with and without gas flow) at the top of the reactor can be easily measured in the absence of inner cylinder rotation, the gas holdup $\left(\varepsilon_{G}\right)$ could also be determined. The results of these measurements are shown in Fig. 10, where it can be seen that gas holdup increases linearly as a function of superficial gas velocity with a slope $u_{G} / \varepsilon_{G}=0.23$, which is essentially the same as the value of 0.24 reported for cylindrical bubble columns [62]. 


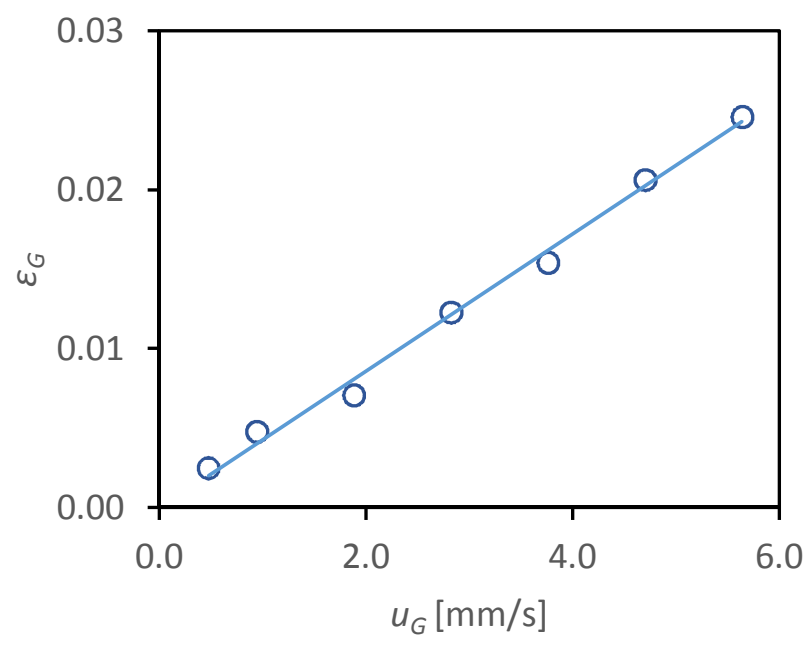

Fig. 10. Measured gas holdup versus gas flow rate for an annular bubble column (no cylinder rotation).

The value for the interfacial surface area can then be calculated using the following equation [53].

$a=6^{\varepsilon_{G}} / d_{32}$

and the usual definition of the Sherwood number can be used [53,63]:

$\operatorname{Sh}=\frac{k_{L} d_{32}}{D_{L}}$

Sherwood numbers computed using Eq. (20) are presented in Fig. 11 and are compared with those obtained in cylindrical bubble column experiments of Akita \& Yoshida [63]. Here the Galilei number is defined as:

$\mathrm{Ga}=\frac{g d_{32}^{3}}{v_{L}^{2}}$

Fig. 11 shows that the Sherwood numbers for the annular bubble column are larger than those reported for cylindrical bubble columns. Fig. 5 may provide insight into the cause of this difference. Specifically, most bubbles in a cylindrical bubble column have low aspect ratio and are essentially spherical. The annular geometry produces many more bubbles with high aspect ratio (greater ellipticity) and consequently greater specific interfacial area. Evidently, the greater specific wall area and narrow confinement in the annular geometry results in increased bubble deformation due to high shear stress near solid surfaces. 


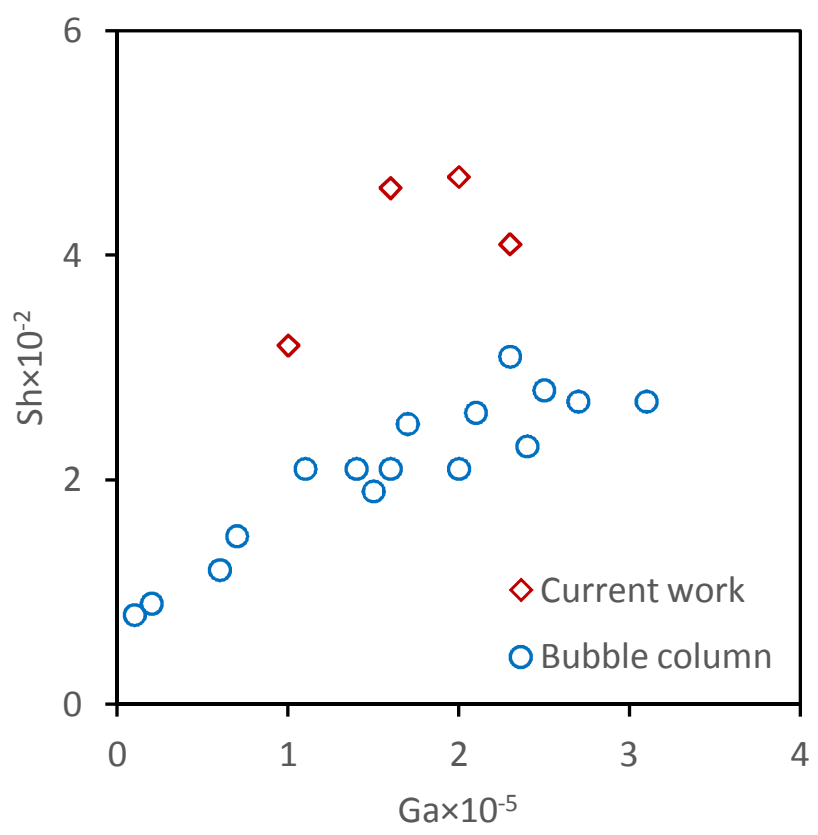

Fig. 11 Comparison of Sherwood vs Galilei numbers for annular and cylindrical bubble columns [49].

\section{Conclusion}

Experimental measurements were performed to obtain gas bubble size and shape distributions and liquid phase volumetric mass transfer coefficients in a vertically oriented semi-batch Taylor-Couette reactor for several combinations of axial and azimuthal Reynolds numbers. Bubble size distributions were found to be lognormally distributed, and the Sauter mean diameter was shown to monotonically increase with increasing inner cylinder rotation speed and gas flow rate according to a power law relation.

Similarly, a correlation was developed to describe the dependence of the liquid side volumetric gas-liquid mass transfer coefficient on azimuthal and axial Reynolds numbers. Although the mass transfer coefficient increases with both axial and azimuthal Reynolds numbers, the dependence upon azimuthal Reynolds number is relatively weak when compared to data obtained in horizontal Taylor vortex reactors with co-flow of gas and liquid. The observed differences can be explained by considering the contribution of the buoyant force to bubble size and spatial distribution. Specifically, in horizontal reactors the buoyant force can be resolved into radial and azimuthal components and does not contribute to axial motion. As a result, increase in the inner cylinder rotation speed (azimuthal Reynolds number) leads to increased azimuthal and radial dispersion and smaller bubble size. In contrast, for vertical Taylor vortex reactors, the buoyant force acts only in the axial direction, and an increase in cylinder rotation speed concentrates bubbles near the inner cylinder wall and between the Taylor vortices, hence producing larger bubbles. 
For the instance of no inner cylinder rotation, the Taylor vortex reactor reduces to an annular bubble column and mass transfer coefficients can be compared with those for traditional cylindrical bubble columns. Bubble size and shape measurements demonstrate that bubbles have higher aspect ratio in annular columns compared to those observed in cylindrical columns, leading to slightly greater values of the volumetric mass transfer coefficient in the annular system.

Furthermore, volumetric mass transfer coefficients in vertically oriented gas-liquid Taylor vortex reactors do not increase as rapidly with increasing azimuthal Reynolds number as in horizontally oriented reactors. Typical values for the turbulent flow conditions studied here ranged between $k_{L} a=0.01-0.02 \mathrm{~s}^{-1}$. When compared with characteristic time scales for slow reaction processes, such as those that occur in photosynthetic microorganisms to produce bio-oil, these values suggest that vertical gas-liquid Taylor vortex reactors are not mass transfer limited for these applications.

\section{Acknowledgement}

The authors acknowledge the efforts of undergraduate lab assistants, Ekoue Kangni and Eric Hessing in designing and performing the experiments. We also gratefully acknowledge financial support for this work from the National Science Foundation (CBET-1236676).

\section{References}

[1] V. Schlüter, W. Deckwer, Gas/liquid mass transfer in stirred vessels, Chem. Eng. Sci. 47 (1992) 23572362. doi:10.1016/0009-2509(92)87060-4.

[2] M. Bouaifi, G. Hebrard, D. Bastoul, M. Roustan, A comparative study of gas hold-up, bubble size, interfacial area and mass transfer coefficients in stirred gas-liquid reactors and bubble columns, Chem. Eng. Process. 40 (2001) 97-111. doi:10.1016/S0255-2701(00)00129-X.

[3] A. Kapic, T. Heindel, Correlating Gas-Liquid Mass Transfer in a Stirred-Tank Reactor, Chem. Eng. Res. Des. 84 (2006) 239-245. doi:10.1205/cherd.05117.

[4] A.J. Ungerman, T.J. Heindel, Carbon monoxide mass transfer for syngas fermentation in a stirred tank reactor with dual impeller configurations, Biotechnol. Prog. 23 (2007) 613-620. doi:10.1021/bp060311z.

[5] L. Labík, R. Vostal, T. Moucha, F. Rejl, M. Kordač, Volumetric mass transfer coefficient in multipleimpeller gas-liquid contactors. Scaling-up study for various impeller types, Chem. Eng. J. 240 (2014) 5561. doi:10.1016/j.cej.2013.11.066. 
[6] P.H. Calderbank, A.C. Lochiel, Mass transfer coefficients, velocities and shapes of carbon dioxide bubbles in free rise through distilled water, Chem. Eng. Sci. 19 (1964) 485-503. doi:10.1016/00092509(64)85075-2.

[7] J.J. Heijnen, K. Van't Riet, Mass transfer, mixing and heat transfer phenomena in low viscosity bubble column reactors, Chem. Eng. J. 28 (1984) B21-B42. doi:10.1016/0300-9467(84)85025-X.

[8] J.M.T. Vasconcelos, J.M.L. Rodrigues, S.C.P. Orvalho, S.S. Alves, R.L. Mendes, A. Reis, Effect of contaminants on mass transfer coefficients in bubble column and airlift contactors, Chem. Eng. Sci. 58 (2003) 1431-1440. doi:10.1016/S0009-2509(02)00675-9.

[9] V. Linek, T. Moucha, M. Kordač, Mechanism of mass transfer from bubbles in dispersions, Chem. Eng. Process. Process Intensif. 44 (2005) 353-361. doi:10.1016/j.cep.2004.05.010.

[10] A. Behkish, R. Lemoine, L. Sehabiague, R. Oukaci, B.I. Morsi, Gas holdup and bubble size behavior in a large-scale slurry bubble column reactor operating with an organic liquid under elevated pressures and temperatures, Chem. Eng. J. 128 (2007) 69-84. doi:10.1016/j.cej.2006.10.016.

[11] G.I. Taylor, Stability of a Viscous Liquid Contained between Two Rotating Cylinders, Philos. Trans. R. Soc. A Math. Phys. Eng. Sci. 223 (1923) 289-343. doi:10.1098/rsta.1923.0008.

[12] P.R. Fenstermacher, H.L. Swinney, J.P. Gollub, Dynamical instabilities and the transition to chaotic Taylor vortex flow, J. Fluid Mech. 94 (1979) 103. doi:10.1017/S0022112079000963.

[13] C.D. Andereck, S.S. Liu, H.L. Swinney, Flow regimes in a circular Couette system with independently rotating cylinders, J. Fluid Mech. 164 (1986) 155. doi:10.1017/S0022112086002513.

[14] R.J. Donnelly, Taylor-Couette flow: The early days, Phys. Today. 44 (1991) 32-39. doi:10.1063/1.881296.

[15] D. Lathrop, J. Fineberg, H. Swinney, Transition to shear-driven turbulence in Couette-Taylor flow, Phys. Rev. A. 46 (1992) 6390.

[16] P.I. Pudjiono, N.S. Tavare, J. Garside, K.D.P. Nigam, Residence time distribution from a continuous Couette flow device, Chem. Eng. J. 48 (1992) 101-110. doi:10.1016/0300-9467(92)85011-W.

[17] S.T. Wereley, R.M. Lueptow, Spatio-temporal character of non-wavy and wavy Taylor-Couette flow, J. Fluid Mech. 364 (1998) 59-80. doi:10.1017/S0022112098008969.

[18] S.T. Wereley, R.M. Lueptow, Velocity field for Taylor-Couette flow with an axial flow, Phys. Fluids. 11 (1999) 3637. doi:10.1063/1.870228.

[19] L. Wang, M.G. Olsen, R.D. Vigil, Reappearance of azimuthal waves in turbulent Taylor-Couette flow at large aspect ratio, Chem. Eng. Sci. 60 (2005) 5555-5568. doi:10.1016/j.ces.2005.05.024. 
[20] M. Bilson, K. Bremhorst, Direct numerical simulation of turbulent Taylor-Couette flow, J. Fluid Mech. 579 (2007) 227. doi:10.1017/S0022112007004971.

[21] S. Dong, Direct numerical simulation of turbulent Taylor-Couette flow, J. Fluid Mech. 587 (2007) $373-$ 393. doi:10.1017/S0022112007007367.

[22] D. Pirrò, M. Quadrio, Direct numerical simulation of turbulent Taylor-Couette flow, Eur. J. Mech. B/Fluids. 27 (2008) 552-566. doi:10.1016/j.euromechflu.2007.10.005.

[23] P.K. Dutta, A.K. Ray, Experimental investigation of Taylor vortex photocatalytic reactor for water purification, Chem. Eng. Sci. 59 (2004) 5249-5259. doi:10.1016/j.ces.2004.07.091.

[24] T. Imamura, K. Saito, S. Ishikura, M. Nomura, A new approach to continuous emulsion polymerization, Polym. Int. 30 (1993) 203-206. doi:10.1002/pi.4990300210.

[25] K. Kataoka, N. Ohmura, M. Kouzu, Y. Simamura, M. Okubo, Emulsion polymerization of styrene in a continuous Taylor vortex flow reactor, Chem. Eng. Sci. 50 (1995) 1409-1416. doi:10.1016/00092509(94)00515-S.

[26] M.W. Davis, E.J. Weber, Liquid-Liquid Extraction between Rotating Concentric Cylinders, Ind. Eng. Chem. 52 (1960) 929-934. doi:10.1021/ie50611a027.

[27] G. Baier, M.D. Graham, E.N. Lightfoot, Mass transport in a novel two-fluid Taylor vortex extractor, AIChE J. 46 (2000) 2395-2407. doi:10.1002/aic.690461208.

[28] M. Kim, K.J. Park, K.U. Lee, M.J. Kim, W.-S. Kim, O.J. Kwon, et al., Preparation of black pigment with the Couette-Taylor vortex for electrophoretic displays, Chem. Eng. Sci. 119 (2014) 245-250. doi:10.1016/j.ces.2014.08.036.

[29] J.G. Sczechowski, C.A. Koval, R.D. Noble, A Taylor vortex reactor for heterogeneous photocatalysis, Chem. Eng. Sci. 50 (1995) 3163-3173. doi:10.1016/0009-2509(95)00176-6.

[30] B. Haut, H. Ben Amor, L. Coulon, A. Jacquet, V. Halloin, Hydrodynamics and mass transfer in a Couette-Taylor bioreactor for the culture of animal cells, Chem. Eng. Sci. 58 (2003) 777-784. doi:10.1016/S0009-2509(02)00607-3.

[31] A.H. Brown, A.G. Fredrickson, R.L. Miller, H.M. Tsuchiya, Hydromechanical method to increase efficiency of algal photosynthesis., Ind. Eng. Chem. Process Des. Dev. 3 (1964) 134-143. doi:10.1021/i260010a008.

[32] B. Kong, J. V Shanks, R.D. Vigil, Enhanced algal growth rate in a Taylor vortex reactor, Biotechnol. Bioeng. 110 (2013) 2140-2149. doi:10.1002/bit.24886.

[33] A. Oasmaa, D.C. Elliott, S. Mu, Quality Control in Fast Pyrolysis Bio-Oil Production and Use, Environ. Prog. 28 (2009) 404-409. doi:10.1002/ep. 
[34] B. Kong, R.D. Vigil, Simulation of photosynthetically active radiation distribution in algal photobioreactors using a multidimensional spectral radiation model, Bioresour. Technol. 158 (2014) 141-148. doi:10.1016/j.biortech.2014.01.052.

[35] R. Campero, R. Vigil, Spatiotemporal Patterns in Liquid-Liquid Taylor-Couette-Poiseuille Flow, Phys. Rev. Lett. 79 (1997) 3897-3900. doi:10.1103/PhysRevLett.79.3897.

[36] R. Campero, R. Vigil, Flow patterns in liquid-liquid Taylor-Couette-Poiseuille flow, Ind. Eng. Chem. Res. 38 (1999) 1094-1098.

[37] G. Baier, M.D. Graham, Two-fluid Taylor-Couette flow with countercurrent axial flow: Linear theory for immiscible liquids between corotating cylinders, Phys. Fluids. 12 (2000) 294. doi:10.1063/1.870308.

[38] X. Zhu, R.D. Vigil, Banded liquid-liquid Taylor-Couette-Poiseuille flow, AIChE J. 47 (2001) 19321940. doi:10.1002/aic.690470905.

[39] D.P.M. Van Gils, S.G. Huisman, G.W. Bruggert, C. Sun, D. Lohse, Torque scaling in turbulent TaylorCouette flow with co- and counterrotating cylinders, Phys. Rev. Lett. 106 (2011) 024502. doi:10.1103/PhysRevLett.106.024502.

[40] D.P.M. van Gils, D. Narezo Guzman, C. Sun, D. Lohse, The importance of bubble deformability for strong drag reduction in bubbly turbulent Taylor-Couette flow, J. Fluid Mech. 722 (2013) 317-347. doi:10.1017/jfm.2013.96.

[41] A. Chouippe, E. Climent, D. Legendre, C. Gabillet, Numerical simulation of bubble dispersion in turbulent Taylor-Couette flow, Phys. Fluids. 26 (2014) 043304. doi:10.1063/1.4871728.

[42] R. Maryami, S. Farahat, M.J. Poor, M.H.S. Mayam, Bubbly drag reduction in a vertical Couette-Taylor system with superimposed axial flow, Fluid Dyn. Res. 46 (2014) 055504. doi:10.1088/01695983/46/5/055504.

[43] R. Hubacz, S. Wroński, Horizontal Couette-Taylor flow in a two-phase gas-liquid system: flow patterns, Exp. Therm. Fluid Sci. 28 (2004) 457-466. doi:10.1016/j.expthermflusci.2003.07.004.

[44] E. Dłuska, S. Wroński, T. Ryszczuk, Interfacial area in gas-liquid Couette-Taylor flow reactor, Exp. Therm. Fluid Sci. 28 (2004) 467-472. doi:10.1016/j.expthermflusci.2003.06.003.

[45] H. Djeridi, C. Gabillet, J.Y. Billard, Two-phase Couette-Taylor flow: Arrangement of the dispersed phase and effects on the flow structures, Phys. Fluids. 16 (2004) 128-139. doi:10.1063/1.1630323.

[46] S. Wroński, R. Hubacz, T. Ryszczuk, Interfacial area in a reactor with helicoidal flow for the two-phase gas-liquid system, Chem. Eng. J. 105 (2005) 71-79. doi:10.1016/j.cej.2004.04.002.

[47] E. Dluska, S. Wronski, R. Hubacz, Mass transfer in gas-liquid Couette-Taylor flow reactor, Chem. Eng. Sci. 56 (2001) 1131-1136. doi:10.1016/s0009-2509(00)00331-6. 
[48] Y.T. Shah, B.G. Kelkar, S.P. Godbole, W.-D. Deckwer, Design parameters estimations for bubble column reactors, AIChE J. 28 (1982) 353-379. doi:10.1002/aic.690280302.

[49] K. Akita, F. Yoshida, Bubble Size, Interfacial Area, and Liquid-Phase Mass Transfer Coefficient in Bubble Columns, Ind. Eng. Chem., Process Des. Dev. 13 (1974) 84-90. doi:10.1021/i260045a015.

[50] M. Polli, M. Di Stanislao, R. Bagatin, E.A. Bakr, M. Masi, Bubble size distribution in the sparger region of bubble columns, Chem. Eng. Sci. 57 (2002) 197-205. doi:10.1016/S0009-2509(01)00301-3.

[51] K. Van't Riet, Review of Measuring Methods and Results in Nonviscous Gas-Liquid Mass Transfer in Stirred Vessels, Ind. Eng. Chem. Process Des. Dev. 18 (1979) 357-364. doi:10.1021/i260071a001.

[52] F. Garcia-Ochoa, E. Gomez, Bioreactor scale-up and oxygen transfer rate in microbial processes: An overview, Biotechnol. Adv. 27 (2009) 153-176. doi:10.1016/j.biotechadv.2008.10.006.

[53] M.K. Popovic, C.W. Robinson, Mass transfer studies of external-loop airlifts and a bubble column, AIChE J. 35 (1989) 393-405. doi:10.1002/aic.690350307.

[54] J. Qiao, R. Deng, C.-H. Wang, Droplet behavior in a Taylor vortex, Int. J. Multiph. Flow. 67 (2014) 132139. doi:10.1016/j.ijmultiphaseflow.2014.08.011.

[55] M. Asgharpour, M.R. Mehrnia, N. Mostoufi, Effect of surface contaminants on oxygen transfer in bubble column reactors, Biochem. Eng. J. 49 (2010) 351-360. doi:10.1016/j.bej.2010.01.010.

[56] K. Muroyama, Y. Oka, R. Fujiki, Transport properties of micro-bubbles in a bubble column, J. Chem. Eng. Japan. 45 (2012) 666-671. doi:10.1252/jcej.12we066.

[57] K. Muroyama, K. Imai, Y. Oka, J. Hayashi, Mass transfer properties in a bubble column associated with micro-bubble dispersions, Chem. Eng. Sci. 100 (2013) 464-473. doi:10.1016/j.ces.2013.03.043.

[58] E.W. Weisstein, Prolate Spheroid, MathWorld--A Wolfram Web Resource. http://mathworld.wolfram.com/ProlateSpheroid.html.

[59] R. Battino, T.R. Rettich, T. Tominaga, The Solubility of Oxygen and Ozone in Liquids, J. Phys. Chem. Ref. Data. 12 (1983) 163-177. doi:10.1063/1.555680.

[60] K. Sujatha, A. Mani, S.S. Murthy, Experiments on a bubble absorber, Int. Commun. Heat Mass Transf. 26 (1999) 975-984.

[61] S. Saenton, T. Illangasekare, Effects of incomplete remediation of NAPL-contaminated aquifers: experimental and numerical modeling investigations, Appl. Water Sci. 3 (2013) 401-414. doi:10.1007/s13201-013-0090-5.

[62] J.H. Hills, The operation of a bubble column at high throughputs: I. Gas holdup measurements, Chem. Eng. J. 12 (1976) 89-99. doi:10.1016/0300-9467(76)87002-5. 
[63] K. Akita, F. Yoshida, Gas Holdup and Volumetric Mass Transfer Coefficient in Bubble Columns. Effects of Liquid Properties, Ind. Eng. Chem. Process Des. Dev. 12 (1973) 76-80. doi:10.1021/i260045a015. 